\title{
Logros de niños y niñas de educación inicial mediante el juego con bloques de Lego \\ Achievements of Early Childhood Education children through play with Lego blocks
}

\author{
*Alix María Casadiego, ${ }^{* *}$ Karina Avendaño Casadiego, ${ }^{*}$ Leidy Carolina Cuervo, ${ }^{* * *}$ Gabriel Avendaño Casadiego \\ *Alvaro Avendaño Rodríguez \\ *Universidad Surcolombiana (Colombia), ${ }^{* *}$ Universidad del Tolima (Colombia), ${ }^{* * *}$ Universidad Nacional de Colombia \\ (Colombia)
}

\begin{abstract}
Resumen. El juego, además de ser una de las experiencias que más disfrutan niños y niñas durante su etapa en educación inicial, les permite aprender y desarrollarse en forma integral. De acuerdo con ello, el presente estudio tiene como objetivo indagar en cuáles logros en relaciones espaciales, temporales y socioafectivas son más exitosos los niños y las niñas de educación inicial e identificar su evolución durante 10 semanas de observación. La metodología tuvo dos fases: inicialmente, mediante la ingeniería didáctica, se construyó un código de observación y una vez construido se realizaron las observaciones durante 10 semanas de trabajo. Las actividades fueron realizadas, durante las horas de juego libre, en las escuelas donde la Facultad de Educación de la Universidad Surcolombiana realiza sus prácticas pedagógicas. Los resultados mostraron que es en la actividad socio afectiva donde se obtienen mayores logros desde el comienzo de la experiencia; por otro lado, el principal logro se obtiene en la característica relación temporal, relacionada con la capacidad para anticiparse a los acontecimientos o predecir resultados, específicamente en la capacidad de organizar un plan para llevar a cabo una idea, que se logra en un 87\%.
\end{abstract}

Palabras claves: Educación inicial, juego, predecir, planear, estrategia.

\begin{abstract}
Playing, in addition to being one of the experiences that children enjoy the most during their stage of early childhood education, allows them to learn and develop in an integral way. In accordance with this, the present study aims to investigate which achievements in spatial, temporal and socio-affective relationships are more successful in early childhood education children and to identify their evolution during 10 weeks of observation. The methodology had two phases: initially, through didactic engineering, an observation code was constructed and once it was ready, observations were made during 10 weeks of work. The activities were carried out, during free play hours, in the schools where the Education Faculty of the Surcolombiana University carries out its pedagogical practices. The results showed that it is in the socio-affective activity where the greatest achievements are obtained from the beginning of the experience; on the other hand, the main achievement is obtained in the characteristic temporal relationship, related to the ability to anticipate events or predict results, specifically in the ability to organize a plan to carry out an idea, is achieved $87 \%$.
\end{abstract}

Keywords: Early Childhood Education, game, predict, plan, strategy.

\section{Introducción}

Los juegos son mecanismos básicos del proceso de socialización fundamentados en las características de la cultura en la que se inscriben (Delgado, Aguilera, Zamora, Oviedo \& Moreno, 2017; Rodríguez, Oliveira, \& Navarro, 2019, citado en Belmonte, Sánchez, Cabrera \&, Bujez, 2019).

El juego tiene su papel protagónico en la educación inicial, dado que potencia el desarrollo de niños y niñas desde las interacciones y relaciones que establecen en la cotidianidad (Ministerio de Educación Nacional, 2014). Los momentos de juego permiten compartir con los

Fecha recepción: 25-04-20. Fecha de aceptación: 02-11-20

Alix María Casadiego

alix.casadiego@usco.edu.co amigos; los estudios de Rodríguez, Pereira, Condessa y Pereira (2020) muestran que al alumnado le gusta el recreo escolar, por ser un momento de diversión, para jugar, descansar y compartir, pero lo consideran demasiado corto para disfrutar de él. Además de socializar, el juego incide de manera positiva en el autoconcepto físico de los escolares como ocurre con los juegos cooperativos (Navarro, Rego, \& García, 2018). Con juegos de construcción cooperativos se desarrollan amistades, ampliando su inteligencia emocional.

El objetivo de la educación en los juegos es desarrollar, no sólo jugadores inteligentes, sino observadores pensantes (Bunker, \& Thorpe, 1982, citado en López, \& del Campo, 2018). Se debe prestar atención a la formación de una actitud relevante para el juego entre niños y niñas en educación inicial y sus maestros, los esfuerzos realizados por los profesionales en esta esfera contribu- 
yen al desarrollo del juego como la actividad más importante del período de educación inicial (Legankova, \& Nedvetskaya, 2016). El juego, como actividad innata del ser humano, constituye una de las formas más claras y evidentes para explicar y justificar la forma de vida de las personas a lo largo de la historia, su versión social nos indica la forma de vivir del hombre, las relaciones con sus semejantes y el entorno (Marin, \& Ribas, 2013, citado en Rodríguez, Pereira, \& Navarro, 2019).

Entre los materiales comunes en las aulas de educación inicial encontramos los bloques para ensamblar. En general, para el Ministerio de Educación Nacional (2016), la exploración del medio posibilita el uso de objetos con variadas posibilidades, cuya evidencia se observa cuando el niño participa en juegos de transformaciones y construcción de juguetes con materiales cotidianos y bloques de construcción. El juego con bloques estructurados en educación inicial, contribuye a mejorar habilidades matemáticas como el reconocimiento de formas, lenguaje matemático y aritmética, (Schmitt, Korucu, Napoli, Bryant, \& Purpura, 2018).

Uno de los juegos de ensamblar que ha acompañado a varias generaciones de niños y niñas ha sido los bloques de lego, el sistema de juego con bloques de lego fue establecido desde 1932, por Ole Kirk Christiansen (Lauwaert, 2008). La compañía de juguetes danesa, brindó a niños y niñas, la oportunidad de diseñar su propio mundo creando réplicas de sus creaciones materiales (Pirrie, 2017). Jugar y aprender con juguetes como lego, puede inspirar a las personas a pensar creativamente y resolver problemas de maneras no tradicionales (Lotts, 2016a).

El juego creativo e interactivo, apoya y proporciona andamiaje a todas las áreas del desarrollo del currículo logrando el equilibrio entre las diferentes áreas del desarrollo del niño (Gil, Romance, \& Nielsen, 2018). Este proceso del desarrollo infantil, se concibe como un entramado biológico, psicológico, social, cultural e histórico, para lo cual se proponen las dimensiones PersonalSocial, Corporal, Comunicativa, Artística y Cognitiva, que deben ser fortalecidas a través de la educación, (Alcaldía Mayor de Bogotá, Secretaría Distrital de Integración Social, 2013). De acuerdo con Wolfgang, Stannard y Jones (2003) los niños y las niñas que tienen experiencias intensivas basadas en el juego y que pueden desempeñarse en altos niveles de construcción con Lego también han mostrado logros matemáticos en entornos escolares formales.

Los procesos de crecimiento y desarrollo del niño, están histórico, social y culturalmente condicionados, por lo tanto, resulta fundamental las condiciones de vida y de educación en las que este proceso transcurre, (Campos, 2009 citado en Pereira, Valladares, Mieres, Velázquez, \& Pichs, 2019). Esas condiciones de vida y de educación, no solo se expresan en la calidad de las condiciones higiénicas y materiales, sino también en un trabajo corresponsable con la familia, donde los aconteceres cotidianos como jugar, explorar, preguntar den cuenta de un acompañamiento intencionado y significativo, (Ministerio de Educación Nacional, 2014). Los ambientes de alta calidad y mejores entornos de aprendizaje en el hogar desarrollan mejores habilidades matemáticas en el primer grado (Lehrl, Kluczniok, \& Rossbach, 2016). El desarrollo de las operaciones prematemáticas es fundamental para abordar conceptos matemáticos en la educación inicial, las estrategias didácticas específicas, es decir enfocadas a lograr un objetivo determinado, deben permitir que niños y niñas se acerquen a conceptos matemáticos complejos, por ejemplo, mediante el desarrollo de habilidades espaciales, introducir el aspecto cardinal y ordinal del número natural (Dinuta, 2013). También el nivel de condición física se asocia a mejores niveles de memoria, cálculo matemático, razonamiento lingüístico y creatividad, (Hidalgo, Ruiz, Loureiro, \& López, 2020).

Cook, Adams, Volden, Harbottle y Harbottle (2011) realizaron un estudio con niños y niñas con discapacidades físicas severas, que consistió en controlar un robot Lego para realizar tareas de juego no estructuradas. Las entrevistas a los maestros revelaron que niños y niñas disfrutaban interactuando con él y demostraban cambios en el comportamiento y las habilidades sociales y de lenguaje después de la interacción, un resultado importante fue el sentido de independencia en el juego y aprendizaje.

Varios estudios sobre el papel de la terapia Lego en las habilidades sociales y la inclusión entre niños, niñas y jóvenes con trastorno del espectro autista, muestra al menos una mejora en la comunicación y habilidades sociales, relaciones familiares, y reducciones en el juego individual (Lindsay, Hounsell, \& Cassiani, 2017).

Barate, Ludovico y Malchiodi (2017) presentan una metodología de enseñanza que mezcla elementos de los dominios de la música y la informática como una clave que permite exponer a los alumnos a los aspectos básicos del pensamiento computacional; la primera fase utiliza Lego ladrillos como una herramienta física y como una metáfora para que los participantes descubran una codificación de notación simple y varios conceptos básicos de la notación musical clásica. 
Aunque Lego tiene diseños exclusivos tanto para niñas como para niños, lo que sugiere que los materiales afirman los estereotipos de género tradicionales, Black, Tomlinson y Korobkova (2016) muestran en sus resultados que Lego trata por igual el potencial constructor de las niñas y de los niños.

Lego cubre un amplio rango de edades, forma parte del material didáctico de preescolares hasta de universidades y bibliotecas, motivo por el cual se han desarrollado varias investigaciones alrededor de este material. En la Biblioteca de Arte de la Universidad de Rutgers, la estación de juegos Lego, ha servido como una herramienta para ayudar a construir una nueva cultura de aprendizaje creativo y ha creado una conexión más fuerte con estudiantes de arte y diseño (Lotts, 2016b). En estudiantes de Ingeniería de Software se incrementó su confianza y compromiso después de Talleres de Construcción de Ciudad Lego (Steghöfer, Burden, Alahyari, \& Haneberg, 2017).

Purcell (2019) sugiere que los métodos creativos son apropiados para abordar problemas complejos en el aula de educación superior; mediante diferentes iteraciones de sesiones reflexivas, en las que se usó Lego para facilitar la participación de estudiantes, destaca cómo los enfoques creativos pueden ayudarles a superar las barreras para su compromiso con un concepto complejo, como la capacidad transformadora del amor profesional dentro de la práctica del trabajo juvenil y comunitario.

Lego también forma parte del material de profesionales, puede usarse para apoyar a estudiantes practicantes que luchan por hacer conexiones entre la teoría y la práctica, además, proporciona a los profesionales formas accesibles de comunicarse cuando intentan involucrar a audiencias diversas con estudiantes y profesionales en entornos de atención social (Cavaliero, 2017).

Además, se usa conjuntamente en universidades e institutos de secundaria, para la implementación de modelos y simulaciones computacionales, aumento de la eficiencia de la educación, diseño y prueba de controladores. Las ideas provenientes del trabajo cooperativo de estudiantes de secundaria y universidad permitieron desarrollar enfoques elementales para enseñar conceptos básicos de control (Fillippov, Fradkov, Ashikhmina, \& Seifullaev, 2010).

La línea de robótica Mindstorms, diseñada para niños y niñas, adquirió estatus universitario en el MIT cuando se interesó por la relación entre la ciencia, la adquisición de conocimiento y el desarrollo de la mente infantil. Lego Mindstorms proporcionó un entorno para la colaboración creativa y emocional de los docentes y estudiantes en Educación de Control (Filippov, Fradkov, \& Andrievsky, 2011). El estudiantado encuentra las sesiones de laboratorio más atractivas y resuelven los problemas con más entusiasmo proponiendo ejercicios prácticos y mejorando los puntajes en sus evaluaciones (Cruz, Fernández, Galindo, González, Stockmans, \& Blanco, 2012). También, el entrenamiento de Lego a partir de bloques codificados por colores, apunta a varios aspectos de interés para los arquitectos facilitando el ambiente de trabajo (Dumitra ${ }^{\circ} \mathrm{cu}$, Corduban, Nica, \& Hapurne, 2014).

Lo expuesto anteriormente puede resumirse en la conjetura de que, al manipular objetos, niños y niñas construyen operaciones lógicas, descubriendo relaciones entre estos objetos; dichas relaciones les permiten organizar, agrupar, comparar, etc, las cuales van expresando a través del lenguaje, la representación, la construcción del número, y la abstracción de las relaciones espaciales y el tiempo, categorías que otorgan sentido al desarrollo del pensamiento lógico matemático en la educación inicial y la primaria.

Estas relaciones y sus expresiones las formularemos en tres aspectos compuestos por: Actividades exploratorias, en donde básicamente observaremos relaciones espaciales de orden, posición y, en general, las producidas al primer contacto con el material; Relaciones temporales, en donde observaremos actividades relacionadas con el manejo del tiempo evidenciadas por ejemplo en la planeación, descripción de sus elaboraciones, entre otras y finalmente los Aspectos socioafectivos, evidenciados en el disfrute de las actividades, expresiones creativas, entre otras.

De acuerdo con estos tres aspectos, los objetivos del presente trabajo son:

1. Indagar en cuáles logros en relaciones espaciales, temporales y socioafectivos son más exitosos los niños y las niñas de educación inicial con el uso de Lego.

2. Identificar la evolución de los niños y las niñas, en el logro de relaciones espaciales, temporales y socio afectivos mediante el juego con bloques de Lego, durante 10 semanas de observación.

\section{Metodología}

La metodología tuvo una fase previa de preparación de las actividades y diseño del instrumento de observación.

A continuación, se describe la muestra y se explica el proceso previo mediante el cual se diseñó el instru- 
mento de observación que sirvió como guía para realizar las actividades en las escuelas donde el programa de Educación Infantil realiza sus prácticas pedagógicas.

\section{Diseño y participantes}

Mediante un código de observación estructurado, construido previamente se describen las observaciones realizadas (Montero, \& León, 2007). Este código de observación, se realizó de acuerdo a la metodología ingeniería didáctica la cual se explica en el siguiente apartado.

Los participantes son niños y niñas de educación inicial pertenecientes a escuelas del municipio de Neiva (Colombia). Los niños y las niñas tienen un rango de edad entre cinco y 5,5 años. Para registrar los avances, se seleccionó una muestra de 174 niños y niñas que fueron los que asistieron con más regularidad a las clases, pues se necesitaba hacer un seguimiento semanal. Los datos del presente artículo corresponden a niños y niñas que completaron las 10 semanas de trabajo.

Las observaciones fueron realizadas por 30 estudiantes de Didáctica del pensamiento matemático, del programa Educación Infantil de la Universidad Surcolombiana y cuenta con la autorización de los responsables de las respectivas instituciones educativas. El criterio de inclusión fue ser alumno de las Instituciones donde la Universidad realiza sus prácticas pedagógicas.

Los centros de práctica son instituciones públicas que tienen convenio con el programa de Educación Infantil de la Universidad Surcolombiana, estos centros tienen un nivel socioeconómico entre medio y bajo, son sectores bastante poblados y los grupos de educación inicial normalmente son de treinta niños y niñas. Las profesoras de estos centros son asesoras de práctica por lo tanto cooperan en todos los procesos de enseñanza de los y las estudiantes practicantes.

\section{Instrumentos y procesos}

\section{Elaboración del instrumento}

El instrumento de observación se elaboró previamente para la asignatura Didáctica de las matemáticas, la metodología para diseñarlo fue la Ingeniería Didáctica. Esta metodología se resume en tres fases: Concepción y análisis a priori de las situaciones didácticas, experimentación y finalmente, análisis a posteriori y evaluación (Campos, 2006).

Concepción y análisis a priori de las situaciones didácticas Es una fase de discusión. En este caso con estudiantes de Didáctica de las matemáticas, conformado por estudiantes del programa Educación infantil de la Universidad Surcolombiana, se analizaron diversos juegos previamente preparados. Durante el desarrollo de la asignatura, las estudiantes realizaban las mismas actividades que serían trabajadas con niños y niñas y, de acuerdo al desempeño de sus compañeras, marcaban también la tabla de observación.

\section{Experimentación}

Inicialmente, cada estudiante hacía ensayos previos con niños y niñas de su entorno para despejar todas las dudas que se pudieran generar con el uso del material. Luego se implementaba el juego con un grupo de 30 niños y niñas de educación inicial. Un grupo de estudiantes daba las instrucciones y participaba con niños y niñas en el juego mientras el otro grupo, se encargaba de registrar las observaciones.

\section{Análisis a posteriori y evaluación}

Una vez registrados los datos, se procedió a discutir con el grupo de observadores las modificaciones necesarias para que la actividad mantuviera la motivación en niños y niñas participantes.

A partir de estas modificaciones se volvía a experimentar hasta que finalmente se seleccionaban las actividades que se realizarían con niños y niñas.

Con los anteriores pasos se creó finalmente el documento de observación.

Una vez diseñado el instrumento se realizó la actividad durante la práctica de la asignatura Didáctica de las matemáticas del semestre 2019-2.

\section{Procedimiento}

Las estudiantes comienzan su práctica pedagógica y realizan la actividad durante la primera hora de clase con niños y niñas. Esta primera hora, corresponde a una sesión denominada juego libre y se caracteriza porque se organizan los materiales a trabajar para que niños y niñas trabajen libremente.

Las practicantes realizaron sus prácticas en grupos. Los niños y las niñas estaban ubicados en mesas hexagonales y a cada practicante le correspondía observar un grupo de 6 niños o niñas.

La profesora, junto con su practicante, se encargaban de explicar las actividades dirigidas como, por ejemplo, realizar una figura, observando una imagen que se colocaba en el tablero, luego niños y niñas procedían a su ejecución mientras la practicante de cada mesa observaba su desempeño. 
A continuación, se describe cada una de las actividades realizadas de acuerdo a la Tabla 1. Instrumento de observación.

Actividades exploratorias: La primera parte de la Tabla 1, se refiere a las Actividades exploratorias, estas actividades se desarrollan inicialmente en forma libre y la idea es observar por ejemplo si el niño arregla objetos en el espacio, explora los materiales, establece diferencias, hace comparaciones, describe posiciones de los objetos y ordena las piezas de mayor a menor número de posibilidades de acople o viceversa. Una vez los niños y niñas se han familiarizado con el material, se realizan juegos de relevos para organizar las fichas de acuerdo a las orientaciones de las practicantes.

Serie en el tiempo - relación temporal: La segunda parte de la Tabla 1, continúa con el aspecto Relación temporal o serie en el tiempo. Se observa si organiza un plan o empieza al azar. Para evaluar si el niño o niña anticipa acontecimientos, se le anima a ver qué pasaría si colocáramos determinada pieza primero. Para esto se propone una figura armada como una pirámide y se le propone retos, como cambiar la posición de acuerdo a otra secuencia, por ejemplo, las más largas arriba, o en el centro y preguntarles qué pasaría, se pregunta cómo organizarlas para conservar el equilibrio, esto permite valorar si H ace predicciones. Una vez logrado, se le pide que describa cómo realizaron la actividad para observar si D escribe acontecimientos en orden. La respuesta a ¿Cuánto falta para terminar? permite puntuar si Reconoce el avance del proyecto.

En cuanto al aspecto Usa patrones típicos repetidos se realizaron las siguientes actividades para comprobar su ejecución:

- Se mezclan fichas de diferentes tamaños, colores y formas para copiar una figura de una imagen fotográfica.

Construir diferentes figuras sencillas con una cantidad específica de fichas de un solo color.

Construir Torre con fichas grandes de cualquier color.

Construir Torre con fichas pequeñas de cualquier color.

Construir Torre con fichas de cualquier tamaño con los colores primarios intercalados.

- Armar Tren con 10 fichas de todos los colores.

Elaborar la Bandera de su región.

En cuanto al ítem Puede recordar y armar figuras pasadas, se le anima a armar figuras ya creadas y se observa si repite la misma secuencia o simplemente inicia una secuencia diferente. Se le presentaba un modelo por unos unos minutos y luego se ocultaba, para que intentara armar la figura sin verla.

Aspecto Socio afectivo: La tercera parte de la Tabla 1 se refiere al aspecto socioafectivo, en este caso, se observaba el disfrute de la actividad, si conversaba sobre la actividad, si creaba personajes diferentes o si construía figuras o escenarios familiares a su entorno.

Tabla 1

Instrumento de 0 bservación

En la siguiente tabla de observación se escriben los nombres de niños y niñas y se escribe "SI" si el niño o la niña, realiza la actividad. (En caso de no mostrar interés se deja en blanco)

nina, realiza la actividad. (En caso deno mostrar interes sedeja en blanco)
\begin{tabular}{|l|c|c|c|c|c|c|c|}
\hline Aspecto & $\begin{array}{c}\text { Nombre } \\
1\end{array}$ & $\begin{array}{c}\text { Nombre } \\
2\end{array}$ & $\begin{array}{c}\text { Nombre } \\
3\end{array}$ & $\begin{array}{c}\text { Nombre } \\
4\end{array}$ & $\begin{array}{c}\text { Nombre } \\
5\end{array}$ & $\begin{array}{c}\text { Nombre } \\
6\end{array}$ \\
\hline Actividades exploratorias & & & & & & \\
\hline Arregla objetos en el espacio & & & & & & \\
\hline Explora los materiales & & & & & & \\
\hline Establece diferencias & & & & & & \\
\hline Hace comparaciones & & & & & \\
\hline Describe posición de los objetos & & & & & & \\
\hline Ordena las piezas de mayor a menor & & & & & & \\
\hline Serie en el tiempo - relación temporal & & & & & & \\
\hline Establece relaciones de tiempo & & & & & & \\
\hline Fundamenta una táctica & & & & & & \\
\hline Organiza un plan & & & & & & \\
\hline Anticipa acontecimientos & & & & & & \\
\hline Describe acontecimientos en orden & & & & & & \\
\hline Reconoce el avance del proyecto & & & & & & \\
\hline Hace predicciones & & & & & & \\
\hline Puede recordar y armar figuras & & & & & & \\
\hline Usa patrones típicos repetidos & & & & & & \\
\hline Arma figuras con las mismas fichas & & & & & & \\
\hline Socio afectivo & & & & & & \\
\hline Crea personajes diferentes & & & & & & \\
\hline Disfruta de la actividad & & & & & & \\
\hline Construye escenarios & & & & &
\end{tabular}

\section{Análisis de datos}

Los análisis de datos se llevaron a cabo mediante las funciones estadísticas del programa Excel. A todo niño o niña se le marcaba cada semana «Sí», sí había logrado cumplir el logro de cada uno de los 19 aspectos. Al final de las 10 semanas, se realizaron los siguientes análisis: Se contabilizó el número de «si» para cada niño o niña, lo cual se expresó en porcentaje con relación al total de «sí» semanal. Se estableció en qué semana obtuvo el mínimo y máximo a lo largo de todo el proceso.

\section{Resultados}

La Tabla 2 presenta las diferentes características y su evolución a lo largo de 10 semanas. Aparece en porcentaje con relación al total de niños y niñas que participaron. Se observa que desde el comienzo niños y niñas obtuvieron logros en las Actividades exploratorias es decir las relacionadas con organización y orden en el espacio. Adicionalmente, estas actividades estuvieron acompañadas de una fuerte actividad física, en la cual, mediante juegos de relevos, los niños y niñas debían ordenar las fichas de acuerdo a instrucciones previas por ejemplo de mayor a menor.

La figura 1 nos presenta la evolución del promedio de eventos exitosos en los tres aspectos estudiados. 
Tabla 2

Evolución Durante 10 Semanas

\begin{tabular}{|c|c|c|c|c|c|c|c|c|c|c|}
\hline \multirow[b]{2}{*}{ Características } & \multicolumn{10}{|c|}{ Semanas } \\
\hline & 1 & 2 & 3 & 4 & 5 & 6 & 7 & 8 & 9 & 10 \\
\hline \multicolumn{11}{|l|}{ Actividades exploratorias } \\
\hline Arregla objetos en el espacio & 73 & 76 & 72 & 71 & 76 & 77 & 75 & 78 & 74 & 85 \\
\hline Explora los materiales & 75 & 75 & 71 & 73 & 76 & 78 & 71 & 74 & 74 & 85 \\
\hline Establece diferencias & 64 & 66 & 69 & 70 & 73 & 75 & 69 & 76 & 74 & 90 \\
\hline Hace comparaciones & 61 & 66 & 64 & 71 & 68 & 66 & 70 & 75 & 72 & 80 \\
\hline Describe posición de los objetos & 48 & 59 & 63 & 62 & 58 & 66 & 65 & 71 & 66 & 80 \\
\hline Ordena las piezas de mayor a menor & 52 & 57 & 62 & 66 & 58 & 58 & 54 & 63 & 67 & 75 \\
\hline \multicolumn{11}{|l|}{ Serie en el tiempo - relación temporal } \\
\hline Establece relaciones de tiempo & 62 & 70 & 72 & 73 & 75 & 69 & 72 & 73 & 66 & 85 \\
\hline Fundamenta una táctica & 49 & 58 & 64 & 65 & 57 & 46 & 54 & 60 & 56 & 80 \\
\hline \begin{tabular}{|l|} 
Organiza un plan \\
\end{tabular} & 52 & 75 & 80 & 87 & 83 & 80 & 76 & 83 & 85 & 85 \\
\hline Anticipa acontecimientos & 53 & 61 & 72 & 72 & 68 & 62 & 67 & 67 & 74 & 85 \\
\hline Describe acontecimientos en orden & 67 & 70 & 72 & 75 & 77 & 74 & 76 & 75 & 81 & 90 \\
\hline Reconoce el avance del proyecto & 73 & 74 & 74 & 81 & 78 & 73 & 71 & 80 & 90 & 100 \\
\hline Hace predicciones & 51 & 52 & 53 & 57 & 63 & 58 & 59 & 65 & 65 & 75 \\
\hline Puede recordar y armar figuras & 49 & 65 & 54 & 57 & 69 & 63 & 61 & 75 & 78 & 80 \\
\hline Usa patrones típicos repetidos & 68 & 66 & 60 & 75 & 74 & 72 & 68 & 86 & 94 & 90 \\
\hline Arma figuras con las mismas fichas & 65 & 71 & 72 & 76 & 75 & 64 & 71 & 70 & 73 & 45 \\
\hline \multicolumn{11}{|l|}{ Socio afectivo } \\
\hline \begin{tabular}{|l|} 
Crea personajes diferentes \\
\end{tabular} & 70 & 73 & 72 & 73 & 75 & 73 & 75 & 77 & 78 & 90 \\
\hline Disfruta de la actividad & 72 & 70 & 70 & 73 & 71 & 71 & 73 & 74 & 75 & 85 \\
\hline \begin{tabular}{|l|} 
Construye escenarios \\
\end{tabular} & 73 & 73 & 71 & 76 & 72 & 72 & 75 & 76 & 72 & 100 \\
\hline
\end{tabular}

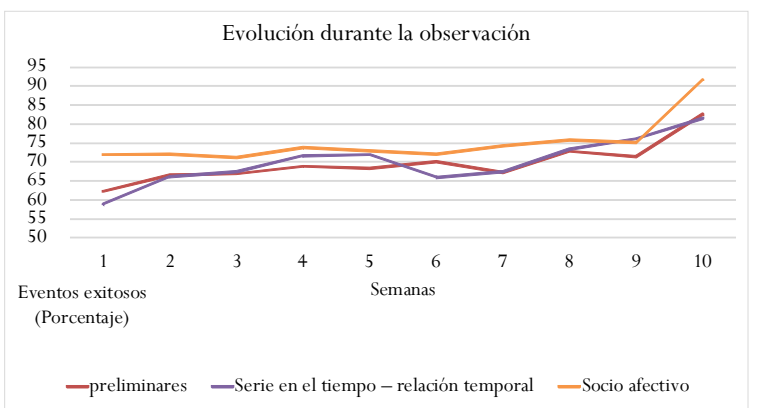

Figura 1. Promedio de eventos existosos en cada

Destaca la parte socio afectiva, como la actividad en la que mayores logros se obtienen desde el mismo comienzo.

La Figura 2 enfatiza que en el aspecto Serie en el Tiempo - relación temporal se logra la mayor variación o cantidad de situaciones exitosas. Es decir, el principal logro del uso de Lego es el desarrollo de la capacidad de organizar un plan para llevar a cabo una idea que lo logra un $87 \%$ en la semana cuatro, le sigue fundamentar una táctica en forma clara en cuanto que empieza en $49 \%$ y termina con un $80 \%$ de niños y niñas que desarrollan el logro en la semana 10.

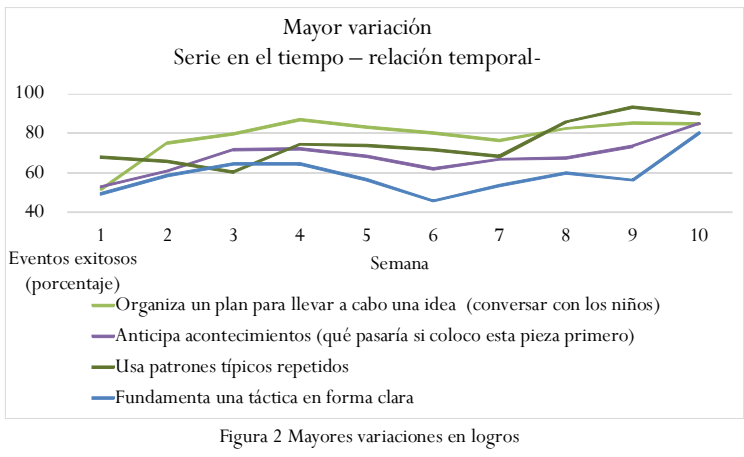

A su vez, en la Figura 3, se observa que en la característica «Actividades exploratorias» alrededor de un $70 \%$ de estudiantes tuvieron éxito desde un comienzo y este logro se mantiene constante durante las primeras nueve semanas, terminando con un pequeño aumento en la semana 10. Las Actividades exploratorias se refieren a Arreglar objetos en el espacio, Explorar los materiales, Establecer diferencias, Hacer comparaciones, Describir posición de los objetos, Ordenar las piezas de mayor a menor número de posibilidades de acople o viceversa.

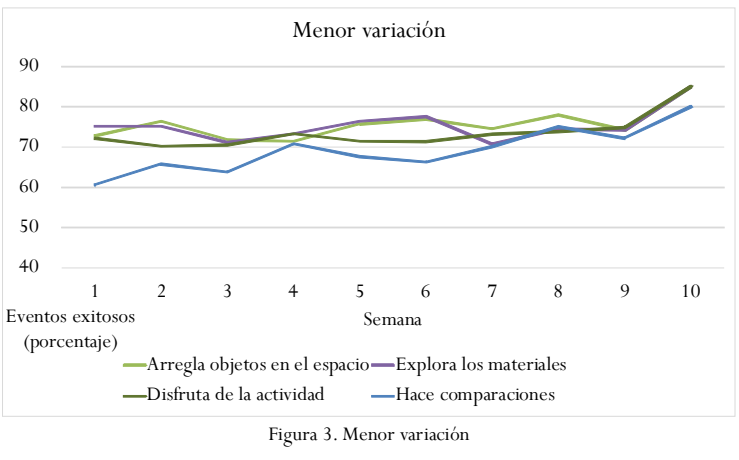

La Figura 4 muestra los mayores logros, es decir, en dónde se obtuvieron los puntajes mayores, no variaciones mayores, que ya expusimos. Puede verse que establecer diferencias, reconocer avances del proyecto, usar patrones repetidos y construir escenarios, formas y figuras familiares mantienen un buen porcentaje de logros a lo largo de todo el proceso, aunque al final, en la semana 10 , hay una elevación vertiginosa en el porcentaje de logros.

Por su parte en la Figura 5, se ilustran las actividades donde, aunque hubo algunos porcentajes de logros buenos como $70 \%$, de todas maneras, fue un resultado sostenido desde la semana uno y con porcentaje bajos con relación a las otras actividades. Como siempre, hacia el final del proceso, semana 10, se evidencia un aumento considerable.

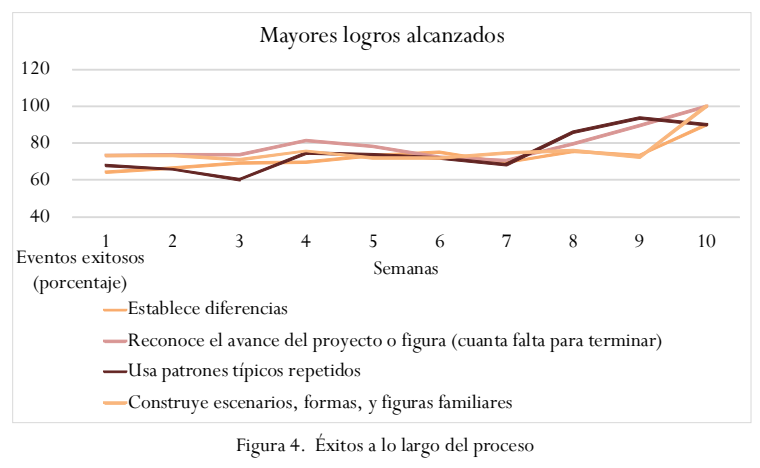




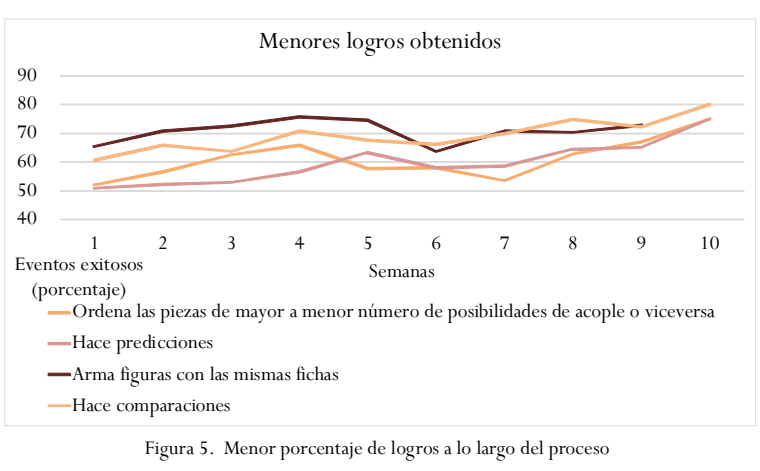

\section{Discusión}

Desde el comienzo de la experiencia los mayores logros del uso de Lego se obtienen en la característica socio afectiva, situación que ya había sido observada en niños y niñas con discapacidades físicas, de acuerdo con Cook et al. (2011) y también, de acuerdo con Lindsay et al. (2017), en niños y niñas autistas en quienes se observaron mejoras en sus habilidades sociales después de la experiencia Lego, y aún para jóvenes estudiantes de Arte y Diseño, como lo afirma Lotts (2016b).

Por otra parte, en cuanto a los avances de niños y niñas mediante el juego con bloques de Lego durante las 10 semanas de observación, en la Figura 2 se muestra que la mayor variación se nota en el desarrollo de la capacidad predictiva en niños y niñas, en planear, anticipar eventos, encontrar patrones repetidos y desarrollar estrategias o tácticas. Esta característica de seriación temporal está relacionada con el desarrollo del pensamiento lógico, como lo confirma el Ministerio de Educación Nacional (2006): el desarrollo del razonamiento lógico empieza en los primeros grados apoyado en los contextos y materiales físicos que permiten percibir regularidades y relaciones; hacer predicciones y conjeturas. Este éxito trae otros logros matemáticos posteriores en entornos escolares formales, como lo investigaron Wolfgang et al. (2003).

Se resalta también que la característica organizar un plan para llevar a cabo una idea, que implica conversar y establecer acuerdos, tuvo un porcentaje de éxito del $87 \%$ en la cuarta semana. Logro que se relaciona con las habilidades de un ingeniero informático para organización de paradigmas de programación o conjunto de métodos sistemáticos aplicables al proceso de diseño de programas (Floyd, 1978, citado en Jiménez et al, 2015).

Finalmente es importante observar que la orientación del juego libre, se convierte en un fuerte aliado en el desarrollo del pensamiento. De acuerdo con Hohmann, Weikart, \& Epstein (2019), mediante la crea- ción de un andamiaje por parte de los adultos, se apoya el actual pensamiento de niños y niñas y los desafían a avanzar en el desarrollo de la creatividad y sus capacidades para razonar y solucionar problemas.

\section{Conclusiones}

En general, el progreso en los tres Aspectos señalados como «Actividades exploratorias», «Relación temporal» $\mathrm{y}$ «Socioafectivo» con el uso de Lego en niños y niñas de educación inicial fue exitoso, pues el 75\% de los eventos tuvieron un porcentaje de éxito comprendido entre $61 \%$ y $81 \%$. La más exitosa fue la característica socio afectiva, en la que se obtienen éxitos sostenidos desde el comienzo de la experiencia.

La actividad organizar un plan para llevar a cabo una idea tuvo un progreso sorprendente, llegando a ser la que más variación tuvo, hasta alcanzar un porcentaje de éxito del $87 \%$. Es decir, es donde más avance se obtiene. Esta característica pertenece a la «Serie en el tiempo - relación temporal», que se relaciona con la capacidad de para anticiparse a los acontecimientos o predecir resultados, la cual fue en general exitosa.

Estos resultados nos dan una guía para darle a las horas del juego, ese papel importante que debe tener en las actividades diarias de niños y niñas. En muchos casos el juego es visto como tiempo perdido, pero hemos observado cómo alrededor de él los niños y las niñas, no solo disfrutan y resuelven problemas de su cotidianidad, sino que fortalecen las relaciones con sus compañeros.

\section{Limitaciones del trabajo y decisiones de ac- ción para la próxima puesta en práctica}

Una de las principales limitaciones de esta investigación es la dificultad para observar el trabajo libre de niños y niñas, pues, a pesar de que cada grupo tenía estudiantes practicantes observando, pueden sentirse intimidados.

Sería interesante ampliar la investigación involucrando a los padres de familia, ya que ellos pueden observar en un contexto más natural y complementar las observaciones.

Es importante también crear la cultura de observar las horas del juego libre, pues en la medida en que orientemos y hagamos un seguimiento a los desempeños de niños y niñas en estos espacios, podremos contribuir en la mejora de las relaciones sociales y el desarrollo general del pensamiento. 


\section{Agradecimientos}

Agradecemos a las estudiantes del semillero de investigación pedagogía del hábito, quienes probaron inicialmente el instrumento y en general a las alumnas y alumnos de Didáctica de las matemáticas y profesoras de los centros docentes que cooperan con el programa Educación infantil.

\section{Referencias}

Alcaldía Mayor de Bogotá, Secretaría Distrital de Integración Social. (2013). Lineamiento pedagógico y curricular para la educación inicial en el Distrito, Bogotá: DVO Universal.

Barate, A., Ludovico, L. A., \& Malchiodi, D. (2017). Fostering computational thinking in primary school through a LEGO ${ }$-based music notation. Procedia Computer Science, (112), 1334-1344. Recuperado de https://doi.org/10.1016/j.procs.2017.08.018

Bateneva, E. V. (2016). Arbitrary memory improvement in older preschoolers using didactic games. ProcediaSocial and Behavioral Sciences, (233), 259-263.

Belmonte, J. L., Sánchez, S. P., Cabrera, A. F., \& Bujez, M. R. V. (2019). Los juegos populares como recurso didáctico para la mejora de hábitos de vida saludables en la era digital. Retos: Nuevas Tendencias en Educación Física, Deporte y Recreación, (36), 266-272.

Black, R. W., Tomlinson, B., \& Korobkova, K. (2016). Play and identity in gendered Lego franchises. International Journal of Play, 5(1), 64-76. Recuperado de https://doi.org/10.1080/ 21594937.2016.1147284

Campos, (2006). Ingeniería didáctica. Cuadernos de investigación y formación en educación matemática, 1(2),19.

Cavaliero, T. (2017). Creative blocs': Action research study on the implementation of Lego as a tool for reflective practice with social care practitioners. Journal of Further and Higher Education, 41(2), 133-142. Recuperado de https:// doi.org/10.1080/0309877X.2015.1070396

Cook, A. M., Adams, K., Volden, J., Harbottle, N., \& Harbottle, C. (2011). Using Lego robots to estimate cognitive ability in children who have severe physical disabilities. Disability and Rehabilitation: Assistive Technology, 6(4), 338-346.Recuperado de https:// doi.org/10.3109/17483107.2010.534231

Cruz-Martín, A., Fernández-Madrigal, J. A., Galindo, C., González-Jiménez, J., Stockmans-Daou, C., \& Blanco-Claraco, J. L. (2012). A LEGO Mindstorms
NXT approach for teaching at data acquisition, control systems engineering and real-time systems undergraduate courses. Computers \& Education, 59(3), 974-988. Recuperado de https://doi.org/10.1016/ j.compedu.2012.03.026

Dinuta, N. (2013). Didactic strategies used in teachinglearninig of premathematical operations in preschool education. Procedia-Social and Behavioral Sciences, (76), 297-301. Recuperado de https://doi.org/10.1016/ j.sbspro.2013.04.116

Dumitra $^{\circ} \mathrm{cu}$, A. I., Corduban, C. G., Nica, R. M., \& Hapurne, T. (2014). LEGO training: An educational program for vocational professions. Procedia-Social and Behavioral Sciences, (142), 332-338. Recuperado de https://doi.org/10.1016/j.sbspro.2014.07.644 Filippov, S. A., Fradkov, A. L., \& Andrievsky, B. (2011, August). Teaching of robotics and control jointly in the university and in the high school based on LEGO mindstorms NXT. In Proceedings of the 18th IFAC World Congress, Milano, Italy (Vol. 28, pp. 9824-9829). Recuperado de https: / / doi.org/10.3182/20110828-6-IT1002.00914

Fillippov, S. A., Fradkov, A. L., Ashikhmina, I. V., \& Seifullaev, R. E. (2010). LEGO mindstorms NXT robots and oscillators in control education. IFAC Proceedings, 43(11), 156-160. Recuperado de https:/ /doi.org/10.3182/20100826-3-TR-4016.00031

Hidalgo, A. M., Ruiz-Ariza, A., Loureiro, V., \& López, E. J. M. (2020). Capacidades físicas y su relación con la memoria, cálculo matemático, razonamiento lingüístico y creatividad en adolescentes. Retos: Nuevas Tendencias en Educación Física, D eporte y Recreación, (37), 473-479.

Hohmann, M., Weikart, D., \& Epstein, A. (2019). La educación de los niños pequeños. Manual de High Scope para los profesionales de la educación infantil, volumen 1. Graciela Borja (Ed.). México: High Scope Press.

Javier, F., Romance, A. R., \& Nielsen, A. (2018). Games and physical activity as indicators of quality in early childhood education. Retos: N uevas Tendencias en Educacion, Fisica, Deporte y Recreacion, (34), 252-257.

Lauwaert, M. (2008). Playing outside the box-on LEGO toys and the changing world of construction play. History and Technology, 24(3), 221-237. Recuperado de https://doi.org/10.1080/ 07341510801900300

Legankova, O. V., \& Nedvetskaya,T. M. (2016). Teacher's professional gaming competence as a condition for preschool education quality enhancement. ProcediaSocial and Behavioral Sciences, (233), 88-90. Recupe- 
rado de https://doi.org/10.1016/ j.sbspro.2016.10.141

Lehrl, S., Kluczniok, K., \& Rossbach, H. G. (2016). Longer-term associations of preschool education: The predictive role of preschool quality for the development of mathematical skills through elementary school. Early Childhood Research Quarterly, (36), 475-488. Recuperado de https: / / doi.org/10.1016/j.ecresq.2016.01.013

Lindsay, S., Hounsell, K. G., \& Cassiani, C. (2017). A

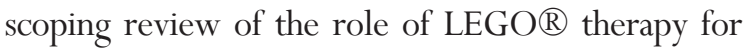
improving inclusion and social skills among children and youth with autism. Disability and $\mathrm{Health}$ Journal, 10(2), 173-182. Recuperado de https: / / doi.org/10.1016/j.dhjo.2016.10.010

López, L. M. G., \& del Campo, D. G. D. (2018). Contributions of the GPET to the GPAI: Tactical context adaptation and game behaviour. Retos: N uevasTendencias en Educación Física, Deporte y Recreación, (34), 323-328.

Lotts, M. (2016a). On the road, playing with Legos $\AA$, and learning about the library: The Rutgers University Art Library Lego Playing Station, Part Two. Journal of Library Administration, 56(5), 499-525. Recuperado de https://doi.org/10.1080/ 01930826.2016.1179517

Lotts, M. (2016b). Playing with LEGO $\AA$, learning about the Library, and «making» campus connections: The Rutgers University Art Library Lego Playing Station, Part One. Journal of Library Administration, 56(4), 359-380. Recuperado de https: / /doi.org/10.1080/ 01930826.2016.1168252

Ministerio de Educación Nacional. (2016) Derechos básicos de aprendizaje. Bogotá: Panamericana Formas e Impresos.

Ministerio de Educación Nacional. (2014). El juego en la educación inicial: Serie de orientaciones pedagógicas para la educación inicial en el marco de la atención integral. Documento No 22. Bogotá: Panamericana Formas e Impresiones.

Ministerio de Educación Nacional. (2006). Estándares básicos en competencias en lenguaje, matemáticas, ciencias y ciudadanas. Recuperado de http: / / goo.gl/lmdfZI.

Ministerio de Educación Nacional (2014). Fortalecimiento institucional para las modalidades de educación inicial. Guía No 54. Bogotá: Panamericana Formas e Impresiones.

Montero, I., \& León, O. G. (2007). A guide for naming research studies in Psychology. International Journal of Clinical and Health Psychology, 7(3), 847-862. Recuperado de http://www.aepc.es/ijchp/ GNEIP07_es.pdf.
Navarro-Paton, R., Rego, B., \& Garcia, M. (2018). Impact of cooperative games on physical self-concept in elementary school students. Retos: N uevas Tendencias en Educacion Fisica, Deporte y Recreacion, (34), 1418.

Pereira, L. G., Valladares, L. R., Mieres, A. A. F., Velázquez, L. M. C., \& Pichs, M. A. E. (2019). Influencia de la estimulación temprana en el desarrollo sensorio-motriz de niños de cuatro a seis años: Una visión desde el Karate Do. Retos: NuevasTendencias en Educación Física, Deportey Recreación, (35), 147 155.

Pirrie, A. (2017). The Lego story: Remolding education policy and practice. Educational Review, 69(3), 271284. Recuperado de https://doi.org/10.1080/ 00131911.2016 .1207614

Purcell, M. E. (2019). Hubris, revelations and creative pedagogy: Transformation, dialogue and modelling 'professional love'with LEGOß. J ournal of Further' and Higher Education, 43(10), 1391-1403. Recuperado de https://doi.org/10.1080/ 0309877X.2018.1490948

Rodríguez, J. E., Pereira, B. O., \& Navarro, R. (2019). Analysis of game and traditional practices in the north zone of the Central Portuguese Way and their relation to educational environment. Retos: N uevas Tendencias en Educacion Fisica, D eportey Recreacion, (35), 25-30.

Rodríguez, J. E., Pereira, V., Condessa, I., \& Pereira, B. (2020). Valor atribuido al recreo escolar por el alumnado de $1^{\circ}$ ciclo de enseñanza básica en Portugal. Retos: N euvas Tendencias en Educacion Fisica, Deporte y Recreacion, 38(38), 188-195.

Schmitt, S. A., Korucu, I., Napoli, A. R., Bryant, L. M., \& Purpura, D. J. (2018). Using block play to enhance preschool children's mathematics and executive functioning: A randomized controlled trial. Early Childhood Research Quarterly, (44), 181-191. Recuperado de https://doi.org/10.1016/ j.ecresq.2018.04.006

Steghöfer, J. P., Burden, H., Alahyari, H., \& Haneberg, D. (2017). No silver brick: Opportunities and limitations of teaching scrum with lego workshops. Journal of Systems and Software, (131), 230247. Recuperado de https://doi.org/10.1016/ j.jss.2017.06.019

Wolfgang, C., Stannard, L., \& Jones, I. (2003). Advanced constructional play with LEGOs among preschoolers as a predictor of later school achievement in mathematics. Early Child Development and Care, 173(5), 467-475. Recuperado de https:// doi.org/10.1080/0300443032000088212 